\title{
AN EVALUATION OF SECURE VANET ENVIRONMENT USING FUZZY APPROACH
}

\author{
Kavita Srivastava \\ Research Scholar, Department of Computer Application, \\ SRM University, Lucknow, India \\ Dr. Bineet Kumar Gupta \\ Associate Professor, Department of Computer Application, \\ SRM University, Lucknow, India
}

\begin{abstract}
Purpose: Vehicular Ad Hoc Networks (VANET) has generally picked up the consideration of the current exam, while ebb is still insufficient to accommodate the north, hostile attacks to meet safety VANET to answer flow network, the driver and to meet the good life and infotainment manufacturer, Try to come to a palatable level.

Methodology: In this paper, we evaluate to different sorts of security factors and difficulties of the VANET was investigated and talked about; we examined a group of forecasts presented similarly fuzzy-based solutions.

Tryouts: VANET security assumes an indispensable part in current time. In this paper a fuzzy strategy on VANET architecture, attacks and their countermeasure utilizing calculation is finished. Additionally, similar investigation, counteraction measure for validation, Non-Repudiation and accessibility and exploration heading characterized for secure VANET network.
\end{abstract}

Key words: VANET Environment, VANET Methodology, Fuzzy approach, Authentication, Non-Repudiation, Availability

Cite this Article: Kavita Srivastava and Bineet Kumar Gupta, An Evaluation of Secure VANET Environment using Fuzzy Approach, International Journal of Management, 11(12), 2020, pp 2237-2244.

http://iaeme.com/Home/issue/IJM?Volume=11\&Issue=12

\section{INTRODUCTION}

In current time, auto collisions are not kidding botheration over the world. Car accidents on Road appraised as ninth principal reason for death. Roughly 1.3 million individuals got kicked the bucket during road incidents just as additional 20 - 50 million are harmed around the world. Some study shows that if the driver gets implication about the mishap still before $1 / 2$ a sec of setback then $60 \%$ of mishaps can be went without. Vehicular Ad hoc Networks 
achieve the explanation through sharing data about road security which related to traffic examination, typical insights like documents and so forth by methods for persistent web affiliation [1, 10]. VANETs are a subgroup of MANETs. In VANETs, vehicles just as roadside foundations are conveying hubs and MANETs highlighting remote correspondence while moving. As of now, there are numerous uses of VANETs which center around various features of transportation associations like driving guide, security of public, assortment of costs assortment, control of traffic on roads, rising security just as expressway framework's power. Quick recovery late in the remote communication network is made conceivable the Inter-Vehicle Communication (IVC) and the road-vehicle communication (RVC) in mobile ad hoc networks (MANETs), which has introduced another type of MANET vehicle known as an ad hoc network (VANET), empower the road well, better driving and the right to information. The present reality is that commuting on roads is like appearing in a battle, and the battle zone lies on the roads, the assessed number of deaths on roads is about 1.2 million individuals yearly around the world, and injured around multiple times of this number, without failing to remember the gridlock that makes leads to so much loss of fuel [2]. VANET security must meet four objectives, it must guarantee that those who have received the data is correct (data genuineness), the source is who he professes to be (message integrity and source validation), the hub sending the message can't be recognized and followed (security) and the framework is vigorous.

\section{VANET SECURITY DOMAINS}

In VANETs, security is needed as VANET parcels (fig 1) holds life basic data and it is basic that these bundles must reach to the drivers with no adjustment or inclusion of information; likewise, the duty of drivers should likewise be perceived that they tell the traffic environment fittingly and inside time [3]. Thus, VANETs must fulfill following security necessities:

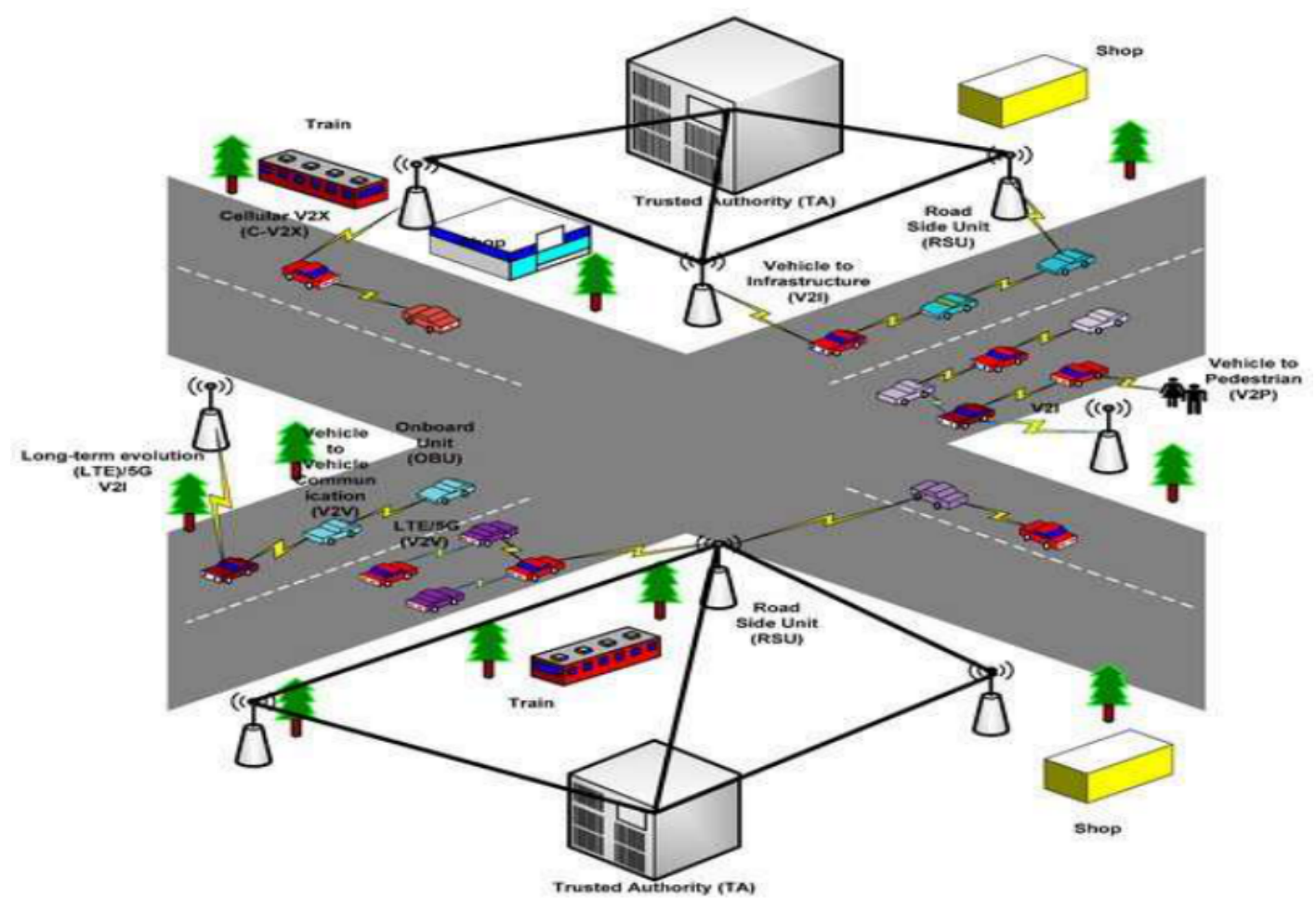

Figure 1 VANET Security Environment [1]

Authentication: It gives us an assurance that information is induced by a genuine customer. It is pivotal that the information which spreads in the living being must be exact and induced 
by a legitimate customer in light of the fact that in VANETs, hubs respond acc. to the information set up from the opposite end.

Non-Repudiation (NR): This dodges cheats from denying their offenses on the grounds that in this regardless of whether the attack happens, NR will assist the capacity to perceive attackers.

Availability: Vehicular networks will require ongoing for some reasons so they should be available constantly. These applications require speedier response from sensor networks or Ad-hoc Network, demolition of the outcome can happen or the back rub can get useless if there is any burglary in seconds for different applications.

\subsection{Various Attacks}

This work primarily focuses on different attacks unleash against protocol itself instead of materialistic security in vehicles. Forswearing of administration attacks This attack; vehicle assets are constrained by the attackers. This sort of attacks additionally forestalls appearance of basic data by sticking the meeting or correspondence medium [4]. The creator, give an answer for conquer this kind of attack by exchanging distinctive correspondence advancements, for example, DSRC, UTRA-TDD [5]. In Replay attack the past data is sent again by the attacker to get the advantage of current situation at the hour of message sending. Fundamental 802.11 gives no protections against this attack because of the nonattendance of extraordinary succession numbers or time stamp. The fundamental rationale of this attack is to deflect vehicles recognizable proof in quick in and out occasion.

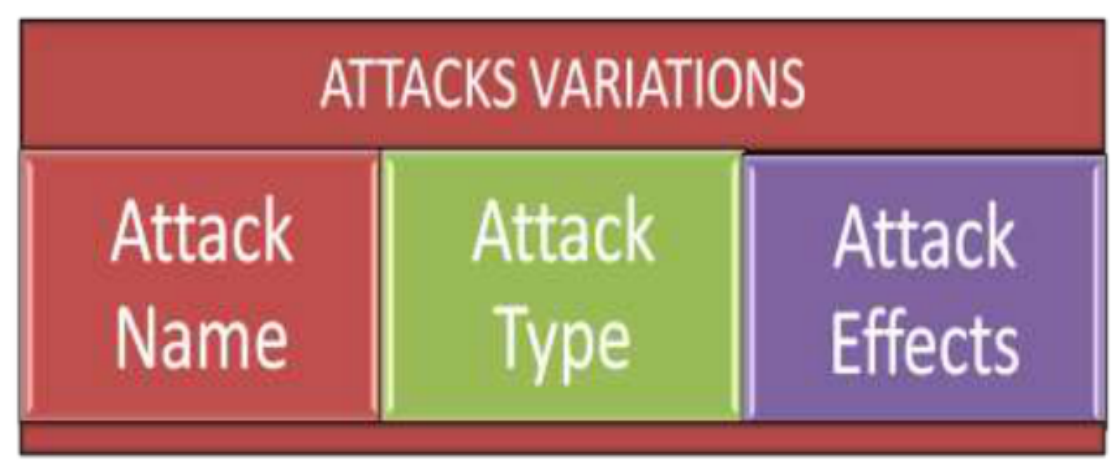

Figure 2 Attack variation and its affect

Attack Name: Impersonation attack, Bogus Information, Malware

Attack Type: Malicious, active, Insider, Network attack

Attack Effects: Availability, Authentication, Non repudiations

\section{PROPOSED MODEL FOR SECURE VANET METHODOLOGY}

VANETs can possibly change the framework. People travel through the development of a safe interoperable remote transportation framework which comprises different vehicles, mobile phones, traffic signals etc [6]. But, VANETs are defenseless to security dangers because of combined reliance upon transmission, registering, and control components. In this way, tying down the start to finish correspondence in VANETs turns into a significant territory of examination. Numerous scientists have proposed a few security protocols so far to improve the honesty, classification, no renouncement, access control, and so on to give secure VANETs to its clients. Along these lines, the general objective of security protocols of VANETs is to perceive pernicious hubs in the network by utilizing reasonable system [7]. 
To do research we will use MATLAB (version13a). It is a High-level language which is utilized for numerical calculation, representation, and application improvement. It has an Interactive domain for iterative investigation, configuration and critical thinking. It gives backing to recreation of TCP, directing, and multicast conventions over all systems remote. It provides support for simulation of TCP, routing, and multicast protocols over all VANETs networks [8].The proposed convention performs in wired and remote systems. In this we will build a adaptive neuro-fuzzy system approach which incorporates a lot of versatile operators in the pursuit space. The adaptive fuzzy system-based approach will have the ability to detect multiple attacks in VANETs by using various adaptive neuro-fuzzy system approaches.

\section{Rules- Set of any two High Parameters show the significantly secure VANET environment}

1. If (Auth is Low_Auth) and (Non_repu is Low_NR) and (Avai is Low_Avail) then (Secure_VANET is Less_Secure) (1)

2. If (Auth is Low_Auth) and (Non_repu is Low_NR) and (Avai is Mod_Avail) then (Secure_VANET is Less_Secure) (1)

3. If (Auth is Low_Auth) and (Non_repu is Mod_NR) and (Avai is Low_Avail) then (Secure_VANET is Less_Secure) (1)

4. If (Auth is Mod_Auth) and (Non_repu is Low_NR) and (Avai is Low_Avail) then (Secure_VANET is Less_Secure) (1)

5. If (Auth is Mod_Auth) and (Non_repu is Mod_NR) and (Avai is Low_Avail) then (Secure_VANET is Modrate_Secure) (1)

6. If (Auth is Mod_Auth) and (Non_repu is Mod_NR) and (Avai is Mod_Avail) then (Secure_VANET is Modrate Secure) (1)

7. If (Auth is Low_Auth) and (Non_repu is Mod_NR) and (Avai is Mod_Avail) then (Secure_VANET is Modrate_Secure) (1)

8. If (Auth is Mod_Auth) and (Non_repu is Low_NR) and (Avai is Mod_Avail) then (Secure_VANET is Modrate_Secure) (1)

9. If (Auth is Mod_Auth) and (Non_repu is Mod_NR) and (Avai is Low_Avail) then (Secure_VANET is Modrate_Secure) (1)

10. If (Auth is High_Auth) and (Non_repu is Mod_NR) and (Avai is Mod_Avail) then

(Secure_VANET is Modrate_Secure) (1)

11. If (Auth is High_Auth) and (Non_repu is High_NR) and (Avai is Mod_Avail) then

(Secure VANET is Highly_Secure) (1)

12. If (Auth is High_Auth) and (Non_repu is High_NR) and (Avai is High_Avail) then

(Secure_VANET is Highly_Secure) (1)

13. If (Auth is High_Auth) and (Non_repu is Mod_NR) and (Avai is High_Avail) then

(Secure_VANET is Highly_Secure) (1)

14. If (Auth is Mod_Auth) and (Non_repu is High_NR) and (Avai is High_Avail) then

(Secure_VANET is Highly_Secure) (1)

\section{ANALYSIS}

By definition, Fuzzy Logic is a utilization of set hypothesis wherein Crisp and Non-Crisp limit sets are broke down for their level of participation as for the mathematical worth comprehended in the semantic terms. Leave $U$ alone the widespread set and $F$ be the fuzzy arrangement of $U$ for security issues. In this way, in fuzzy set $F$, every component can be planned to $[0,1]$ by utilizing the enrollment capacities. $\mu \mathrm{F}: \mathrm{U} \rightarrow[0,1]$ And $\mu \mathrm{F}(\mathrm{u})$ can be deciphered as the level of participation of component $u$ in fuzzy set $F$ for every $u \neg U$. Participation work in fuzzy logic speaks to the level of truth in dubiously characterized sets and furthermore permits us to graphically speak to a fuzzy set dependent on which patterns, edges can be discovered, that can be utilized to take the basic choices when various covering factors are affecting a specific cycle.. So in fuzzy set F, every component can be planned to 
$[0,1]$ by utilizing the participation capacities. $\mu \mathrm{F}: \mathrm{U} \rightarrow[0,1]$ And $\mu \mathrm{F}(\mathrm{u})$ can be deciphered as the level of enrollment of component $u$ in fuzzy set $F$ for every $u \neg U$. Participation work in fuzzy logic speaks to the level of truth in security factors characterized sets and furthermore permits us to graphically speak to (figure 6,7 and 8) a fuzzy set dependent on which patterns, edges can be discovered, that can be utilized to take the basic choices when different covering factors are affecting a specific cycle. Fuzzy logic has been utilized to actualize the surface diagram in different relationship with security issues.

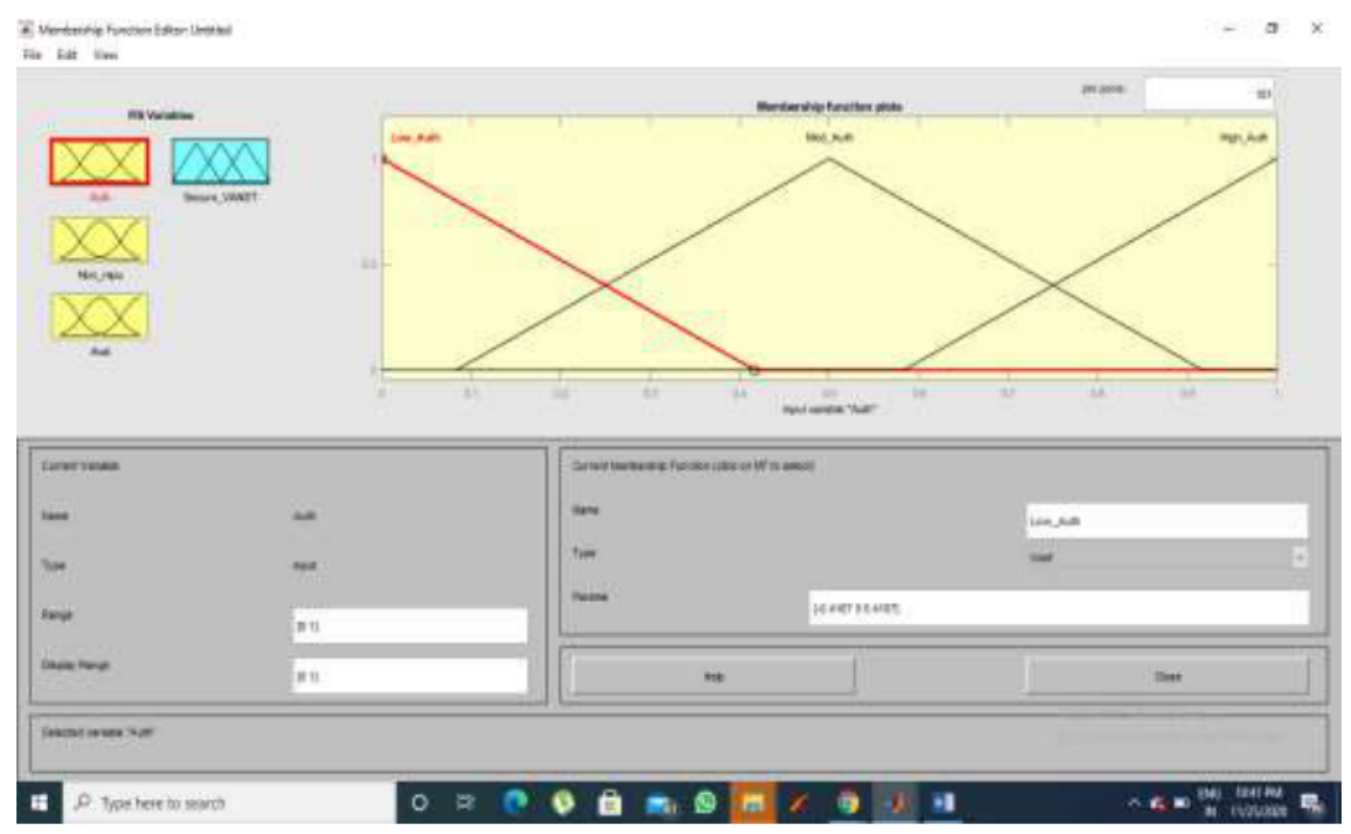

Figure 3 Authorization_Range_Chart

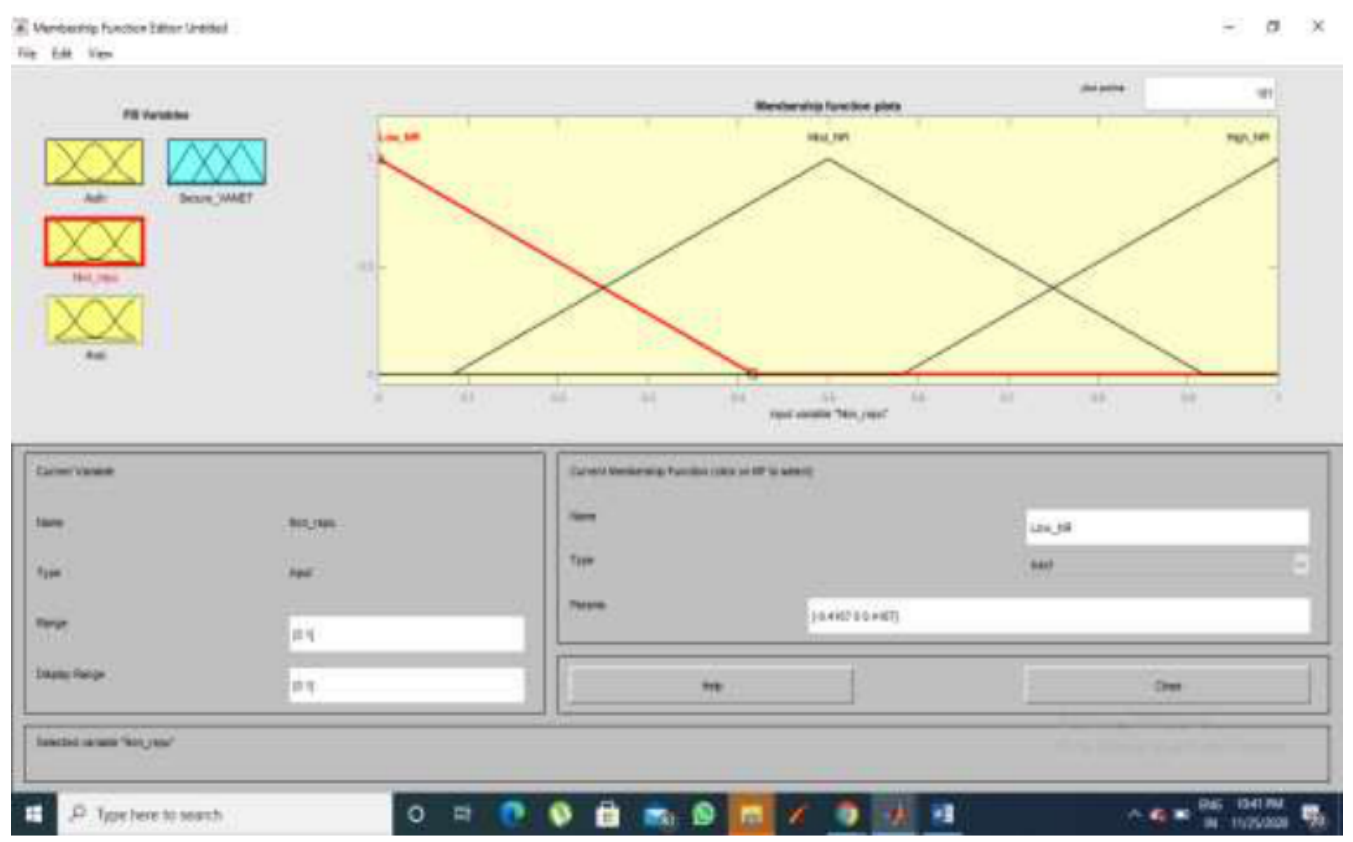

Figure 4 Non-repudiation Range Chart 


\section{Kavita Srivastava and Bineet Kumar Gupta}

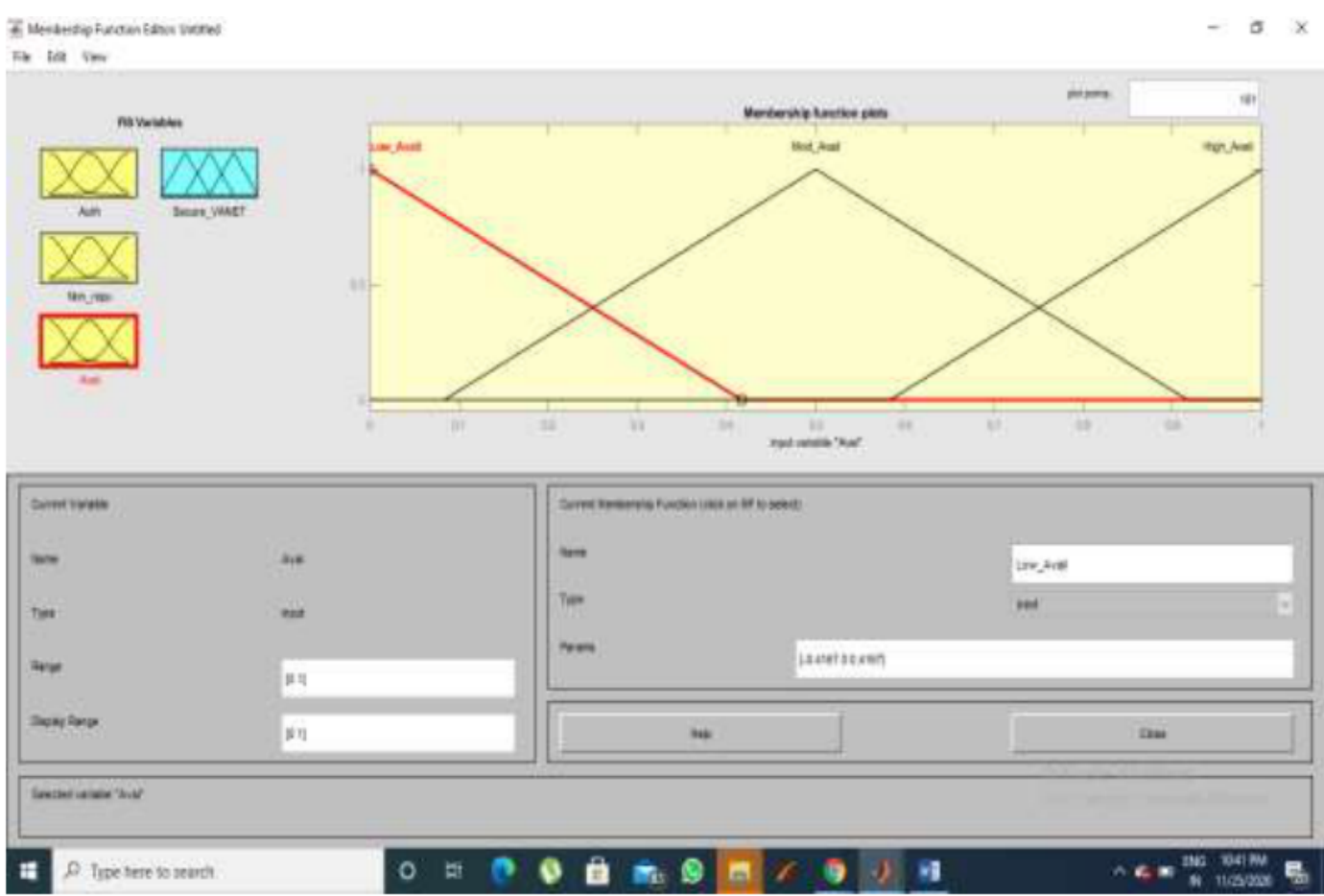

Figure 5 Availability Range Chart

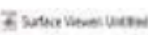

$\Rightarrow$ tee vis ane.
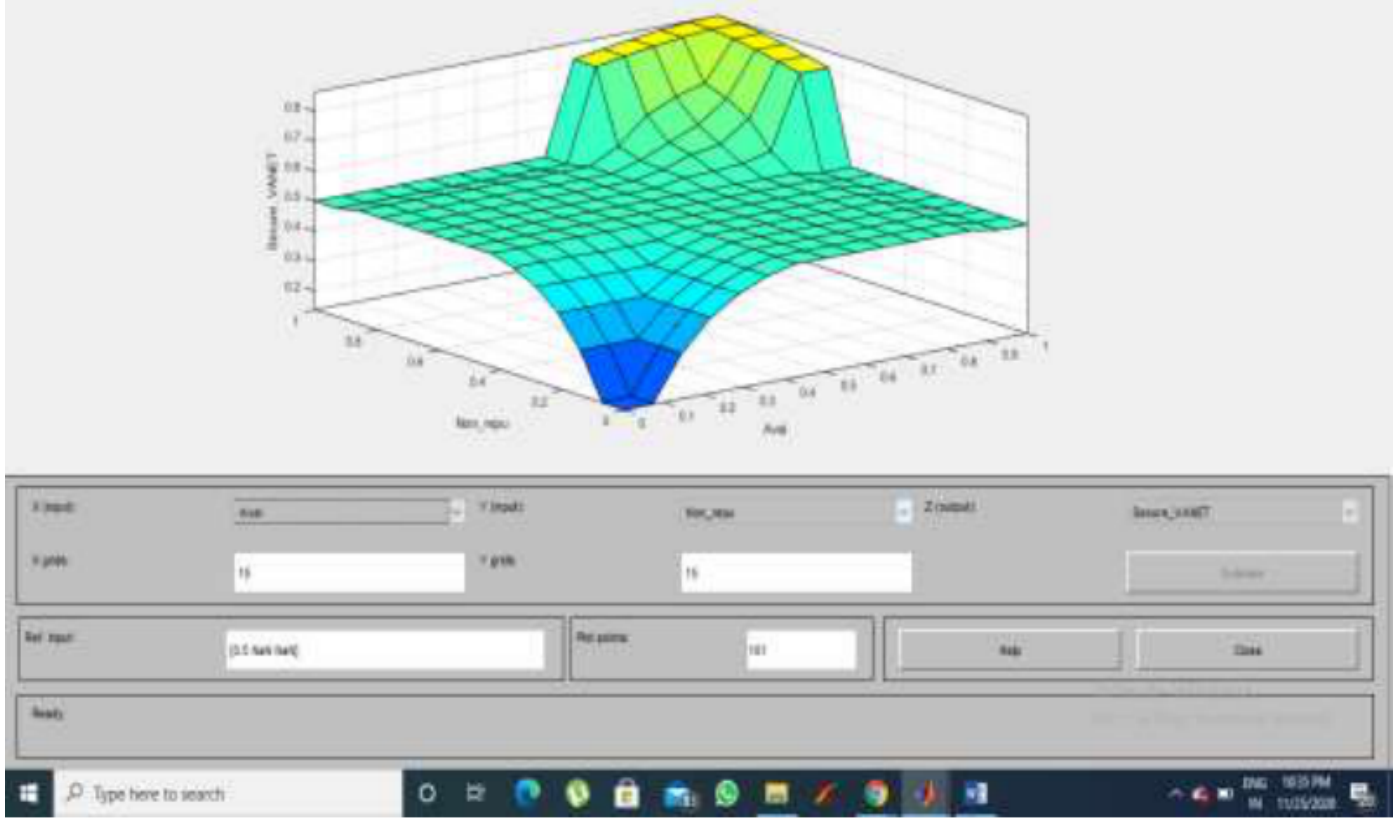

Figure 6 Surface Curve of Non-Repudiation and Availability 


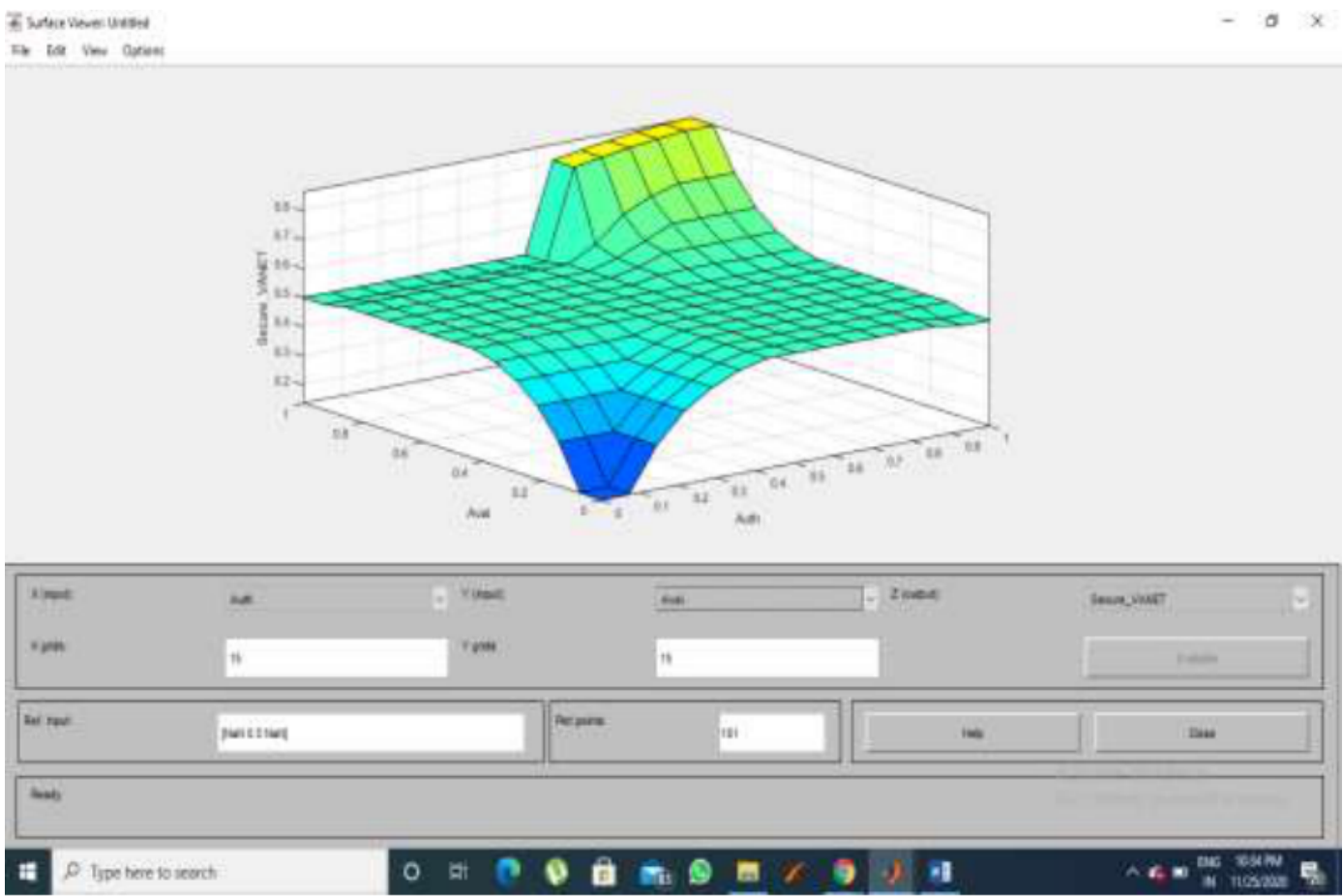

Figure 7 Surface Curve of Authorization and Availability

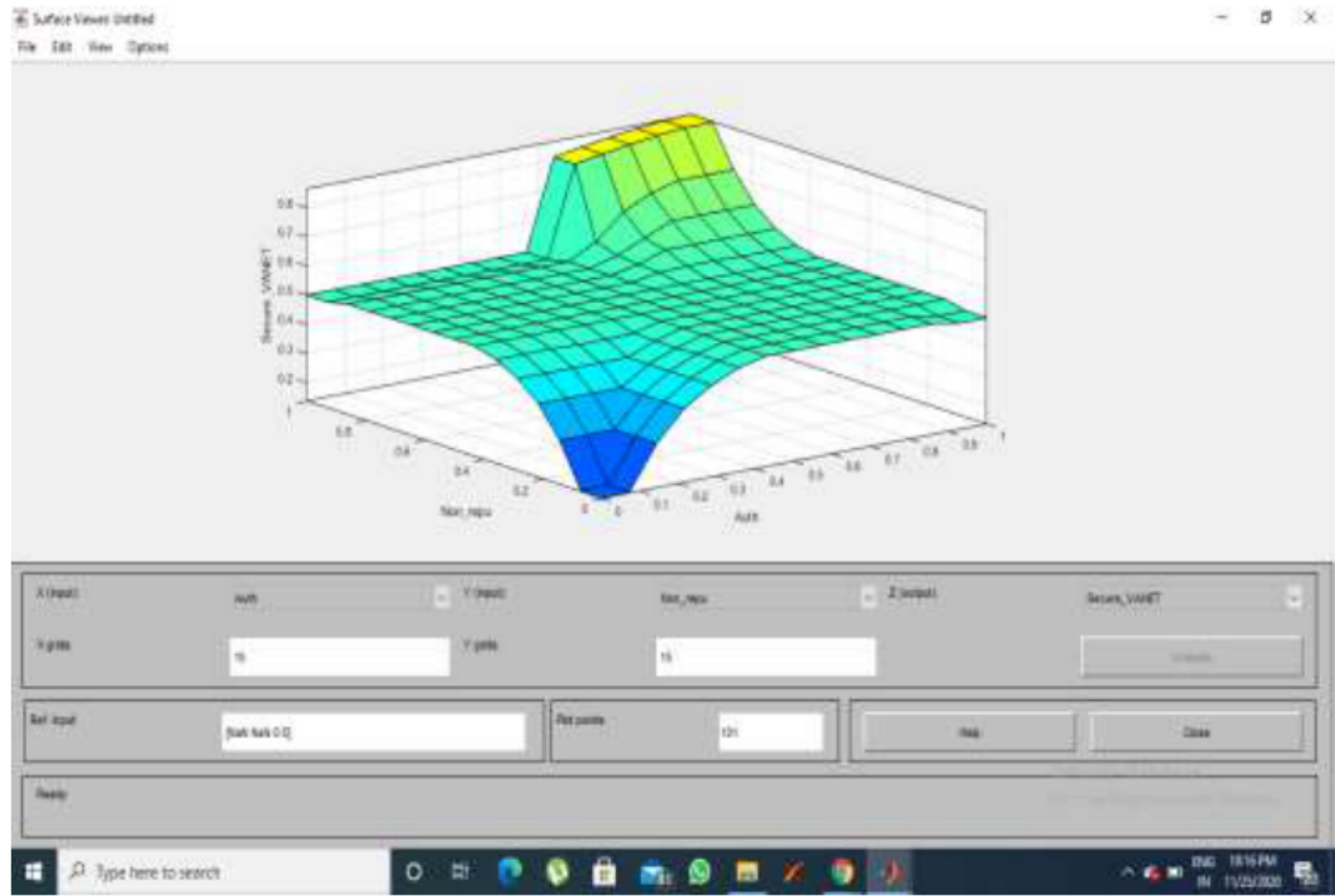

Figure 8 Surface Curve of Authentication and Non-repudiation

\section{DISCUSSION \& CONCLUSION}

In this paper, we have dissected the presentation of the calculation in three angles: validation, non-disavowals and accessibility. Security issues is a thorough assessment file under multifaceted thought. It is the norm to check the exhibition of the calculation or algorithm. Normal reach is the security volume, which is the primary factor to guarantee the familiarity of a client vehicle's information demands. The quantity of solicitation disappointments is the 
occasions that the specialist vehicle can't give total information transmission to the client vehicle, which shows the soundness of information transmission. We have examined the effect of the different kinds of protections on the different issues, normal throughput, number of solicitation disappointments, and normal utilization during the time spent cooperation among client and specialist vehicles. Figure 6 shows that the security parameters relationship bend got by the fuzzy based calculation is higher than different issues while changing the quantity of client vehicle demands.

\section{REFERENCES}

[1] https://www.mdpi.com/1424-8220/19/16/3589

[2] Lu, N.; Cheng, N.; Zhang, N.; Shen, X.; Mark, J.W. Connected Vehicles: Solutions and Challenges. IEEE Internet Things J. 2014, 1, 289-299.

[3] S. Goudarziet al., "A systematic review of security in vehicular Ad Hoc network," in Proc. 2nd Symp. WSCN, 2013, pp. 1-10.

[4] R. G. Engoulou, M. Bellaïche, S.Pierre, and A. Quintero, "VANET security surveys," Comput. Commun., vol. 44, pp. 1-13,May 2014.

[5] R. Mishra, A. Singh and R. Kumar, "VANET security: Issues, challenges and solutions," International Conference on Electrical, Electronics, and Optimization Techniques, 2016.

[6] J. M. de Fuentes, A. I. Gonzalez-Tablas and A. Ribagorda, "Overview of security issues in vehicular ad-hoc networks," Handbook of Reseach on Mobility and Computing, IGI Global, 2010 .

[7] M. De Fuentes, A. I. González-Tablas, and A. Ribagorda, "Overview of security issues in vehicular Ad-hoc networks," in Handbook of Researchon Mobility and Computing: Evolving Technologies and Ubiquitous Impacts, M. Cruz-Cunha and F. Moreira, Eds. Hershey, PA, USA: IGI Global, 2011, pp. 894-911

[8] I. Bhardwaj and S. Khara, "An Analytic Study of Security Solutions for VANET," International Journal of Computer Applications, Vol. 132, No.10, Dec.2015

[9] O. Abumansoor and A. Boukerche, "A secure cooperative approach form online-of-sight location verification in VANET,' 'IEEE Trans. Veh. Tech-nol., vol. 61, no. 1, pp. 275-285, Jan. 2012.

[10] R. A. Shaikh and A. S. Alzahrani, "Intrusion aware trust model for vehicular Ad hoc networks,'’ Secur. Commun. Netw, vol. 7, no. 11,pp. 1652-1669, 2014. 